\title{
EWLA - Ein unverzichtbares Juristinnen-Netzwerk in Europa
}

Prof. Dr. Beate Rudolf, Freie Universität Berlin, EWLAVizepräsidentin

\section{Was ist und wozu brauchen wir EWLA?}

„Europa ist ein integraler Bestandteil unseres Lebens als Frauen und als Juristinnen geworden. Deshalb bin ich Mitglied bei EWLA." - Mit diesen Worten fasst Dr. h.c. Renate Jaeger, Richterin am Europäischen Gerichtshof für Menschenrechte, die zwei zentralen Zielsetzungen der Europäischen Juristinnenvereinigung EWLA (European Women Lawyers' Association) zusammen: EWLA ist ein Netzwerk von und für Juristinnen in Europa. Hier treffen sich Richterinnen, Rechtsanwältinnen, juristische Praktikerinnen aus Wirtschaft, Verwaltung und Nichtregierungsorganisationen sowie Wissenschaftlerinnen zum Erfahrungsaustausch, Netzwerken und zur Politikberatung aus Juristinnensicht. Was der djb auf nationaler Ebene in Deutschland ist, ist EWLA auf europäischer Ebene - nur noch nicht so lange: EWLA wurde erst im Jahr 2000 gegründet. Der djb war daran maßgeblich beteiligt - am Gründungskongress in Berlin nahmen zahlreiche djb-Mitglieder teil und unter ihrer ersten Präsidentin, der deutschen Rechtsanwältin Elisabeth Kotthaus (geb. Müller), entwickelte sich EWLA schnell zu einer ernstzunehmenden Akteurin auf europäischer Ebene. Auch betätigte sich EWLA als Geburtshelferin für Juristinnenvereinigungen in den neuen Mitgliedstaaten der EU.

Mitglieder von EWLA sind nämlich neben vielen Juristinnen aus den Mitgliedstaaten der Europäischen Union und der Staaten des Europäischen Wirtschaftsraums (EWR - Norwegen, Island und Liechtenstein) sowie der Schweiz auch nationale Juristinnenvereinigungen aus diesen Staaten. Der djb wird beispielsweise durch Ministerialrätin Sabine Overkämping vertreten, die im September erneut von der Mitgliederversammlung des djb zur Delegierten des djb bei EWLA gewählt wurde. Alle - individuelle wie korporative Mitglieder eint das Bestreben, persönliche Kontakte unter Juristinnen in Europa aufzubauen, den fachlichen Austausch untereinander zu stärken und auf Rechtsentwicklungen auf europäischer Ebene Einfluss zu nehmen. Dabei geht es insbesondere, aber nicht ausschließlich, um die Verwirklichung der Geschlechtergleichstellung und um die Wahrung der europäischen Grundrechte. So hat EWLA bei- spielsweise auf die Verhandlungen zum (gescheiterten) europäischen Verfassungsvertrag und auf die Ausgestaltung des Vertrages von Lissabon eingewirkt. Unter der Ägide der zweiten Präsidentin, der finnischen Wirtschaftsjuristin Leena Linnainmaa, hat EWLA sich nachdrücklich für eine verbesserte Repräsentation von Frauen in Aufsichtsräten und Entscheidungsgremien in Wirtschaft und Politik eingesetzt. Auch nach ihrem Ausscheiden aus dem Präsidentenamt wirkt Leena Linnainmaa weiterhin für EWLA im Netzwerk der Kommission „Frauen in Entscheidungspositionen" mit.

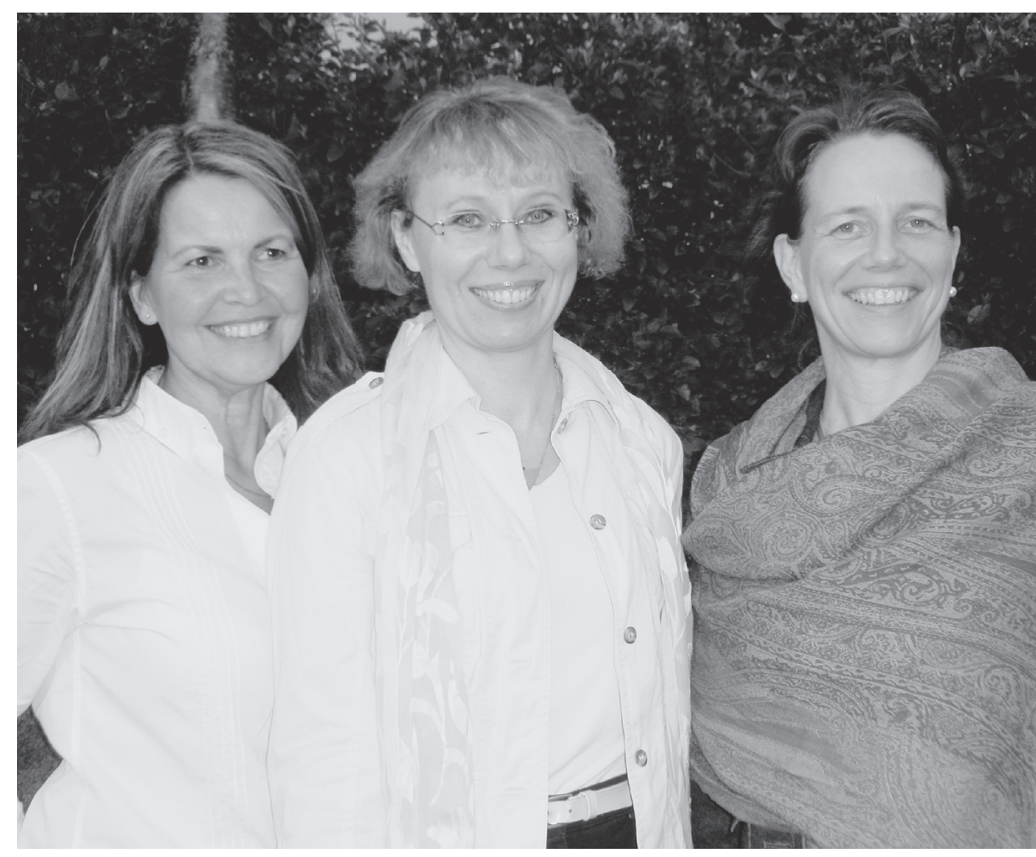

$\Delta$ Die bisherigen EWLA-Präsidentinnen (v.l.n.r.): Herdís Thorgeirsdóttir (Island, seit 2009), Leena Linnainmaa (Finnland, 2005-2009), Elisabeth Kotthaus (Deutschland, 2000-2005).

\section{Bewährtes Instrument zum Netzwerken: Die jährlichen EWLA-Kongresse}

Netzwerken geht nicht ohne persönliche Kontakte - daher führt EWLA seit ihrer Gründung alljährlich an wechselnden Orten in Europa Kongresse durch. Hier lassen sich neue Kontakte knüpfen und bestehende Verbindungen auffrischen, und dies stets mit einem vielfältigen Programm und hochkarätig besetzten Podien. Das Programm reicht traditionell von Themen zu Gleichstellung und Antidiskriminierung über grundrechtliche Fragen und solche der institutionellen Entwick- 
lung der EU bis hin zum Handels- und Wirtschaftsrecht, Familienrecht, Strafrecht und der Juristenaus- und -fortbildung. Für viele Teilnehmerinnen ist es eine willkommene Mischung aus Vertiefung eigener Schwerpunkte, mit besonderem Fokus auf europarechtlichen Einflüssen, und allgemeiner europarechtlicher Fortbildung, für die im Berufsalltag oft zu wenig Zeit bleibt.

So bot etwa der diesjährige Kongress in Reykjavík, der unter dem Titel „Fundamental Rights and Financial Markets“ stand, eine Paneldiskussion mit der französisch-norwegischen Ermittlungsrichterin Eva Jolie, die über die praktischen Schwierigkeiten bei der Strafverfolgung von Finanzinstitutionen in Island berichtete, und Workshops über Unternehmensverantwortung sowie über die Auswirkungen der Finanzkrise auf Familien. Ein anderer Workshop setzte sich kritisch mit der Fortentwicklung des europäischen Antidiskriminierungsrechts auseinander, welches nunmehr das Verbot der Geschlechtsdiskriminierung gegenüber anderen Diskriminierungsverboten zurückfallen lässt. Leider ein „Dauerbrenner“ auf EWLA-Kongressen ist das Thema häusliche Gewalt; angesichts der vielfältigen innovativen Strategien in anderen europäischen Staaten sind diesbezügliche Workshops für deutsche Juristinnen erfahrungsgemäß von großem Nutzen. Die Wirtschaftsjuristinnen befassten sich mit Fragen des Risikomanagements. Soweit möglich arbeiten Workshops Resolutionen aus, die in der Mitgliederversammlung diskutiert und verabschiedet werden. Sie legen die Positionen von EWLA zu den behandelten Themen fest und bestimmen damit die Lobby-Arbeit von EWLA bei europäischen Institutionen im laufenden Jahr.

Stellungnahmen zu aktuellen Rechtsentwicklungen wurden bisher vom EWLA-Vorstand, in dem nach Möglichkeit alle Mitgliedstaaten vertreten sind, erarbeitet. In der Zukunft sollen verstärkt die Arbeitsgruppen eingebunden werden, in denen sich die EWLA-Mitglieder nach fachlichen Schwerpunkten zusammengefunden haben. Gegenwärtig werden diese Arbeitsgruppen neu konstituiert, um möglichst weitgehend die Bereiche gesetzgeberischer Aktivitäten auf EUEbene abzudecken. Alle bisherigen Resolutionen und Stellungnahmen finden sich auf der EWLA-Website <www.ewla. org >.

\section{Neu: 〈www.ewla.org〉 als Kommunikationsforum und Infor- mationsquelle}

Mit dem eingangs wiedergegebenen Zitat von Renate Jaeger präsentiert sich auch die neue Website von EWLA. Die Website soll dazu beitragen, dass EWLA in Zukunft ein noch besseres, weil kontinuierliches Netzwerk für Juristinnen in Europa ist. Dies ist das besondere Anliegen der in diesem Jahr neu gewählten Präsidentin, der isländischen Professorin
Herdís Thorgeirsdóttir. Als ehemalige Journalistin bringt sie die idealen Voraussetzungen dafür mit, die Website lebendig und attraktiv zu machen. Die Website bietet Nachrichten zu aktuellen Entwicklungen auf europäischer Ebene und zu frauenrechtsrelevanten Themen in der EU und ihren Mitgliedstaaten, Informationen über Mitglieder sowie über Aktivitäten von EWLA und nationalen Juristinnenvereinigungen. Für EWLA-Mitglieder besteht die Möglichkeit, über eigene Aktivitäten zu berichten und damit auf sich und/oder eigene Veröffentlichungen aufmerksam zu machen. Veröffentlichungen von EWLA-Mitgliedern haben zudem eine eigene Rubrik. Für beides genügt eine Mail an <info@ewla. org>. In der Rubrik „Spotlight“ wird zudem allmonatlich ein EWLA-Mitglied vorgestellt; Interessentinnen können sich bei<president@ewla.org> melden. Gegenwärtig noch im Aufbau begriffen ist das Angebot, Kontaktdaten von juristischen Institutionen (z.B. Kammern), Wissenschaftlerinnen an juristischen Fakultäten und Anwältinnen in Europa durch simplen Mausklick zu erhalten. Diese Möglichkeit, europaweit für sich zu werben, können auch Nichtmitglieder von EWLA nutzen; das „Spotlight“ bleibt freilich EWLA-Mitgliedern vorbehalten.

\section{0: Kongress zum zehnjährigen Jubiläum von EWLA}

Im kommenden Jahr feiert EWLA ihr zehnjähriges Bestehen. Der Jahreskongress soll unter Mitwirkung hochrangiger Persönlichkeiten am 3. und 4. Juni 2010 in Brüssel stattfinden. Er bietet die Gelegenheit, über die Zukunft der europäischen Integration nachzudenken, Ideen zur erhöhten Repräsentation von Frauen in europäischen Führungspositionen zu entwickeln und Schwerpunkte für die künftige Tätigkeit von EWLA zu setzen. Hier können die EWLA-Mitglieder ihre Vorstellungen einbringen und Bedürfnisse artikulieren. Die Kongresseinladung wird Anfang März an die Mitglieder verschickt; das Programm wird auch auf der Website veröffentlicht. Für EWLA-Mitglieder sind die Teilnahmegebühren reduziert; bei Veranstaltungen mit begrenzter Kapazität haben EWLA-Mitglieder Priorität.

\section{Fazit}

Die Mitgliedschaft bei EWLA lohnt sich für jede Juristin, unabhängig von einer europäischen Ausrichtung ihrer Tätigkeit. EWLA bietet persönliche Kontakte zu Kolleginnen und Austausch über die Grenzen hinweg, juristische Fortbildung sowie kritische Teilhabe am „Projekt Europa“ - ob als aktives Mitglied einer Arbeitsgruppe oder „nur“ als europäische Bürgerin durch Unterstützung von EWLA. Eine Anmeldung ist online unter <www.ewla.org > oder per Fax oder Brief möglich (Formulare sind auf der Website erhältlich). EWLA freut sich auf Sie! 